OPEN ACCESS

Edited by:

Anju Devianee Keetharuth, The University of Sheffield,

United Kingdom

Reviewed by:

Richard Xu,

Hong Kong Polytechnic University,

Hong Kong, China

Sophie Williams,

University of Derby, United Kingdom

*Correspondence:

Harpal S. Randeva

harpal.randeva@warwick.ac.uk

loannis Kyrou

kyrouj@gmail.com

tThese authors have contributed equally to this work and share senior authorship

Specialty section:

This article was submitted to

Quality of Life,

a section of the journal

Frontiers in Global Women's Health

Received: 03 January 2021 Accepted: 12 May 2021

Published: 04 June 2021

Citation:

Kite C, Atkinson L, McGregor G,

Clark CCT, Brown JE, Kyrou I and Randeva HS (2021) Sleep Disruption and Depression, Stress and Anxiety

Levels in Women With Polycystic Ovary Syndrome (PCOS) During the Lockdown Measures for COVID-19 in

the UK.

Front. Glob. Womens Health

2:649104.

doi: 10.3389/fgwh.2021.649104

\section{Sleep Disruption and Depression, Stress and Anxiety Levels in Women With Polycystic Ovary Syndrome (PCOS) During the Lockdown Measures for COVID-19 in the UK}

\author{
Chris Kite ${ }^{1,2,3}$, Lou Atkinson ${ }^{3,4}$, Gordon McGregor ${ }^{5,6,7}$, Cain C. T. Clark ${ }^{3,8}$, \\ James E. Brown ${ }^{2,9}$, loannis Kyrou ${ }^{3,6,9,10 * t}$ and Harpal S. Randeva ${ }^{3,9,10 * t}$
}

${ }^{1}$ Centre for Active Living, University of Chester, University Centre Shrewsbury, Shrewsbury, United Kingdom, ${ }^{2}$ School of Biosciences, College of Health and Life Sciences, Aston University, Birmingham, United Kingdom, ${ }^{3}$ Warwickshire Institute for the Study of Diabetes, Endocrinology and Metabolism (WISDEM), University Hospitals Coventry \& Warwickshire National Health Service (NHS) Trust, Coventry, United Kingdom, ${ }^{4}$ School of Psychology, College of Health and Life Sciences, Aston University, Birmingham, United Kingdom, ${ }^{5}$ Department of Cardiopulmonary Rehabilitation, Centre for Exercise \& Health, University Hospitals Coventry \& Warwickshire National Health Service (NHS) Trust, Coventry, United Kingdom, ${ }^{6}$ Centre for Sport Exercise \& Life Sciences, Coventry University, Coventry, United Kingdom, ${ }^{7}$ Warwick Clinical Trials Unit, Warwick Medical School, University of Warwick, Coventry, United Kingdom, ${ }^{8}$ Centre for Intelligent Healthcare, Coventry University, Coventry, United Kingdom, ${ }^{9}$ Aston Medical Research Institute, Aston Medical School, College of Health and Life Sciences, Aston University, Birmingham, United Kingdom, ${ }^{10}$ Division of Translational and Experimental Medicine, Warwick Medical School, University of Warwick, Coventry, United Kingdom

Background: Lockdown measures have been enforced globally in response to the COVID-19 pandemic. Given the comorbidity burden in women with polycystic ovary syndrome (PCOS), these lockdown measures may have a particularly negative impact on sleep health, quality of life (QoL), and depression/stress levels in this population. The aim of this study was to explore whether such potential problems were present in women with PCOS during the COVID-19 lockdown in the UK.

Methods: UK women with PCOS were recruited through social media into a cross-sectional study during the COVID-19 lockdown. The study survey was delivered online, and included demographic and COVID-19 relevant questions, as well as validated questionnaires/scales, namely the Insomnia Severity Index (ISI), Depression Anxiety and Stress Scale (DASS-21), and PCOSQOL questionnaire.

Results: Three hundred and thirty-three women with PCOS [median age: 30.0 (9.0) years] were recruited. Participants were dichotomized based on responses regarding the impact of COVID-19 restrictions on their sleep [negative $(N=242)$ vs. no/positive $(N=91)$ impact]. No differences were noted between groups regarding age, time since PCOS diagnosis, body mass index, or number of comorbidities. Based on the ISI, $44.2 \%$ of participants reporting a negative impact on sleep exhibited at least moderately severe clinical insomnia. Compared to those who reported no/positive effect on sleep, the participants reporting a negative impact on sleep also reported poorer QoL, based on the total PCOSQOL score, with a greater impact of PCOS and poorer mood in the corresponding PCOSQOL domains. Based on the DASS-21, the latter also had 
statistically higher depression and stress levels compared to the former. Finally, for this cohort significant inverse correlations were noted between the ISI and PCOSQOL scores (total and domain scores), whilst the DASS-21 and ISI scores were positively correlated (all $p$-values $<0.001$ ).

Conclusion: The majority of recruited UK women with PCOS reported that the COVID-19 lockdown had a negative impact on their sleep, which was also associated with impaired QoL and higher depression/stress levels. Whilst further research is required, women with PCOS should be considered a vulnerable population that may experience an adverse impact on sleep, QoL and mental health well-being due to lockdown measures during the COVID-19 pandemic.

Keywords: polycystic ovary syndrome, COVID-19, lockdown, sleep, anxiety, depression, stress, quality of life

\section{INTRODUCTION}

Coronavirus disease 2019 (COVID-19), caused by the severe acute respiratory syndrome coronavirus-2 (SARS-CoV-2), usually manifests as a respiratory tract infection with mild symptomatology (asymptomatic in many cases) (1-3). However, COVID-19 can also lead to severe manifestations in a proportion of high-risk individuals with respiratory and/or extra-pulmonary symptoms/complications requiring hospitalization (1-3). As the latter may require intensive care unit (ICU) support and may even be fatal, the COVID-19 pandemic has resulted in enforcement of varying degrees of nationwide lockdown, quarantine and self-isolation measures in many countries worldwide $(4,5)$. These measures aim to reduce SARS-CoV-2 transmission in the general population, and thus the risk of severe COVID-19 in vulnerable groups (e.g., older individuals and patients with certain respiratory and cardio-metabolic diseases) (6-12). Indeed, compelling evidence strongly indicates that certain chronic cardio-metabolic diseases, including diabetes and obesity, constitute key risk factors predisposing to severe COVID-19 (6-12). Notably, although severe COVID-19 is more common in men $(13,14)$, the aforementioned cardio-metabolic comorbidities, which significantly increase the risk of adverse COVID-19 related clinical outcomes, are also markedly prevalent in women with polycystic ovary syndrome (PCOS) (15-17).

PCOS is the most common endocrine disorder in reproductive-aged women, affecting up to $15-20 \%$ of this female population depending on the studied population and the applied diagnostic definition $(18,19)$. After excluding other endocrinopathies with similar symptomatology (19, 20), PCOS is typically diagnosed based on the presence of at least two out of three diagnostic criteria, namely ovulatory dysfunction, hyperandrogenism (clinical and/or biochemical) and polycystic ovaries (PCO) as identified by ultrasound (21). In addition, women with PCOS are also at a high risk of cardio-metabolic complications, particularly obesity, insulin resistance, type 2 diabetes (T2DM), hypertension, non-alcoholic fatty liver disease (NAFLD), and obstructive sleep apnoea (OSA) (22-27). Furthermore, women with PCOS often exhibit psychological comorbidity, with higher prevalence of coexisting anxiety and/or depression (28-31), which also tends to impair their overall quality of life (QoL) compared to women without PCOS (32, 33). Given this increased comorbidity burden, women with PCOS may experience a particularly negative impact from the lockdown/quarantine and self-isolation measures imposed against the transmission of SARS-CoV-2 and may also be at increased risk of severe COVID-19 (16).

Overall, global measures to control the COVID-19 pandemic are expected to inevitably have a negative psychological effect upon the general population (34) due to various factors, including the enforced quarantine measures (35) and their socio-economic impact (36), as well as the concern about COVID-19 (34). This has been previously reported in relation to SARS, which was shown to promote increased stress, anxiety and depression in the general population (37). Such findings are increasingly reported during the current COVID-19 pandemic, with an observed increase in self-reported symptoms of anxiety, depression, and stress (38). Moreover, these negative psychological effects are likely linked to disrupted sleep quality (39). In previous longitudinal studies, new onset mental health issues have been linked to increased sleep disruption (40-42), whilst disturbed sleep is also considered to be a contributing factor to the development of new mental health disorders (43). Of note, irrespective of the COVID-19 pandemic, sleep disturbances are more prevalent in women with PCOS than in the general population $(44,45)$, potentially due to coexisting OSA, particularly in women with poorer metabolic profiles (26), and/or depression which is also a major predictor of poor sleep quality (46). Furthermore, reduced self-esteem and body satisfaction, which are both frequently associated with PCOS, have also been demonstrated to contribute toward disrupted sleep (44).

In this context, it is likely that a 2 -fold effect of increased psychological distress due to the COVID-19 pandemic alongside the established disease-related burden of PCOS may further contribute to impaired sleep quality and QoL, linked also to increased anxiety, depression, and stress levels (47). To date, there have been no published studies exploring these issues in women with PCOS during the COVID-19 pandemic. Therefore, the aim of the current study was to determine whether the COVID-19 pandemic has had a negative effect upon the sleep quality of women with PCOS in the United Kingdom (UK), and whether any such impairment was associated with 
reduced QoL and increased stress, anxiety, or depression in this female population.

\section{METHODS}

For the purposes of this study, we conducted a cross-sectional study based on a web-based survey between 2 nd June and 17th August 2020. Ethical approval was granted by the ethics committee of Coventry University (application number: P106195) in May 2020. Recruitment was conducted through social media and with the support of Verity (the UK PCOS charity) and PCOS support groups on Facebook. Participant eligibility criteria included female sex with a previous medical diagnosis of PCOS, age from 18 to 45 years, and UK residency.

A range of structured and validated questionnaires were used to collect the study data; questionnaires were completed online using the survey software, Qualtrics ${ }^{\odot} \mathrm{XM}$ (Qualtrics XM, Provo, Utah, USA). Where participants expressed an interest in participation, a study URL link to the survey was emailed to them directly. Alternatively, participants could access the questionnaires directly via the same study URL posted on social media channels. The study URL link contained an initial participant information sheet, as well as provision of informed consent. Where participants failed to complete the provided online survey, this was translated as withdrawal from the study and all data for these participants were excluded from the final analyses.

To capture relevant participant characteristics, a demographics questionnaire was created for this survey. This included self-reported information about time since PCOS diagnosis and diagnosed PCOS phenotype, height, weight, age, questions relating to sleep affected by COVID-19 restrictions (i.e., "to what extent do you believe that quarantine measures due to COVID-19 have affected your sleep pattern?"), presence of comorbidities and typical sociodemographic questions. In the context of this study, the PCOSQOL was utilized as a validated measure of QoL (48). The PCOSQOL is a diseasespecific questionnaire which was developed and validated to measure QoL in UK women with PCOS, and is also the first PCOS-specific measurement tool to encompass all phenotypic subgroups according to the most recent diagnostic criteria (48). Briefly, this questionnaire incorporates 35 Likert-based questions allowing participants to report the impact of various PCOSrelated symptoms upon their day-to-day life, with subscales which allow reporting of the impact of PCOS, infertility, hirsutism, and mood upon QoL (49-51). Total scores are summated with lower scores indicative of poorer QoL.

Information about the mental health well-being of each participant was also captured using the Depression, Anxiety and Stress Scale (DASS-21) (52). The DASS-21 includes 21 questions, seven relating to each domain for depression, anxiety and stress, and requires respondents to rate their level of agreement (0-3) to a series of statements. Each domain score is calculated by summing the responses and multiplying by two, whilst normative and cut-points for depression, anxiety, and stress are provided. Finally, the Insomnia Severity Index (ISI) was applied to measure participants' self-perceived insomnia (53), as a validated and reliable tool for the assessment of insomnia severity $(54,55)$. The ISI targets the subjective symptoms and consequences of insomnia, as well as the degree of concerns or distress caused by those difficulties. The ISI is composed of seven items that, respectively, evaluate the severity of sleep-onset (initial), sleep maintenance (middle), early morning awakening problems (terminal), satisfaction with current sleep pattern, interference with daily functioning, noticeability of impairment attributed to the sleep problem, and level of distress caused by the sleep problem. Each of these items is rated on a five-point Likert scale and the time interval is "in the last 2 weeks." Total ISI scores range from 0 to 28 , with higher scores indicating greater insomnia severity.

\section{Statistical Analysis}

Statistical analysis was completed in IBM SPSS Statistics for Windows (Version 26.0, IBM Corp; Armonk, NY) and in R statistical software [(56), using the car: and MASS: packages] (57, 58), and statistical significance was set at $p<0.05$. Descriptive characteristic reports were generated and ShapiroWilk tests of normality were completed. Accordingly, a nonparametric approach was adopted for subsequent analysis, as appropriate. Responses to the question "to what extent do you believe that quarantine measures due to COVID19 have affected your sleep pattern?" were split into a dichotomous response-namely, into negative effects or no effect/positive effects-which was used as a categorical variable. Independent samples Mann-Whitney $U$-tests were completed to evaluate the between group differences. Spearman's correlations between the ISI and other questionnaires (i.e., PCOSQOL total and domain scores, and the DASS-21) were also completed for the entire study cohort and also within each group.

Given the Likert scale nature of the data in this study, where Likert scales are a special case of ordinal data, we utilized an ordinal logistic regression (OLR) approach. The OLR approach is comparable to a conventional multiple regression approach, where there may be one dependent variable and one or more independent variables. It does however differ from ordinary least squares multiple regression, by treating the dependent variable as an ordered categorical variable, based upon the principle of cumulative-odds (59). The coefficient of determination, $R^{2}$, summarizes the proportion of variance in the dependent variable associated with the independent variables, with larger $R^{2}$ values indicating that more of the variation is explained by the model, to a maximum of 1 . However, in non-parametric regression, it is not possible to compute a traditional $R^{2}$, and a pseudo $R^{2}$ is computed instead. In this study, we opted to report Nagelkerke's $R^{2}\left(\mathrm{R}_{\mathrm{N}}^{2}\right)$, which is an adjusted version of the Cox \& Snell $R^{2}$ that adjusts the scale of the statistic to cover the full range from 0 to $1(60,61)$. To test the statistical significance of each model coefficient $(\beta)$, we used the Wald test to compute a Wald statistic with a chi-square distribution. All participant background characteristics were adjusted for in the OLR. 


\section{RESULTS}

In total, 333 participants met the eligibility criteria and consented to participate in the present study, completing the online survey. Pertinent demographics/characteristics for the recruited study cohort are presented in Table 1 . The vast majority of the study participants $(92.5 \%)$ were of White ethnic background. Of the recruited cohort, $40 \%$ were married and a further $40 \%$ were single, with the remaining $20 \%$ being in other non-married relationships, divorced, separated or widowed. Approximately $73 \%$ of participants stated that they have no children. Moreover, the majority $(63.1 \%)$ of the study participants were in fulltime employment, $40.2 \%$ were educated to at least degree level, and $51.1 \%$ had the lowest household income ( $\leq £ 39,999$ ). Finally, participants were asked to self-report their diagnosed PCOS phenotype; $46.5 \%$ indicated that they had all three PCOS diagnostic characteristics (i.e., hyperandrogenism, menstrual disruption, and PCO), 13.5\% PCO and menstrual disruption, 9.6\% PCO and hyperandrogenism, 6.9\% hyperandrogenism and menstrual disruption, and the remaining $23.1 \%$ were unsure of the PCOS phenotype with which they had been diagnosed.

When participants' responses to the question about the impact of COVID-19 restrictions on their sleep were dichotomized into negative and no/positive responses, 242 participants reported that they had experienced either a significant or small negative effect upon their sleep, whilst the remaining 91 reported either no such effect, or at least a small positive effect upon their sleep. Using the ISI scoring guidelines, $44.2 \%$ of those reporting negative effects met the scoring threshold $(>14)$ for a diagnosis of at least moderately severe clinical insomnia. Key outcomes of interest were compared between these two study groups and these findings are summarized in Table 2.

Overall, there were no differences between the study groups regarding age, weight, body mass index (BMI), time since PCOS diagnosis, or number of comorbidities. As expected, the selfreported insomnia severity assessed by the ISI was significantly higher in those who reported a negative effect of COVID-19 on their sleep quality compared to those who reported no such effect or a relevant positive effect (Table 2). Furthermore, the former also reported poorer QoL, as measured by the total PCOSQOL score, whilst they also reported a greater impact of PCOS and poorer mood in the corresponding PCOSQOL domains. Finally, based on the corresponding DASS-21 scale scores, those reporting a negative effect of COVID-19 on their sleep quality also had statistically higher depression and stress levels, but not anxiety, compared to those who reported no such effect (Table 2).

For the entire study cohort, Spearman's correlation tests showed an inverse correlation between the ISI and PCOSQOL total score $\left(r_{\mathrm{s}}=-0.384, p<0.001\right)$, and domain scores for Impact of PCOS $\left(r_{\mathrm{s}}=-0.379, p<0.001\right)$, Infertility $\left(r_{\mathrm{s}}=-0.225, p<0.001\right)$, Hirsutism $\left(r_{\mathrm{s}}=-0.205, p<0.001\right)$, and Mood $\left(r_{\mathrm{s}}=-0.405, p<0.001\right)$. Furthermore, significant positive correlations were also noted between the ISI and the DASS-21 Depression $\left(r_{\mathrm{s}}=0.377, p<0.001\right)$, Anxiety $\left(r_{\mathrm{s}}=0.410\right.$, $p<0.001)$, and Stress $\left(r_{\mathrm{s}}=0.467, p<0.001\right)$ scores. These correlations between the ISI and the total PCOSQOL score, all PCOSQOL domains apart from Hirsutism, and DASS-21 scores
TABLE 1 | Breakdown of socioeconomic and ethnicity characteristics of interest for the study cohort of UK women with polycystic ovary syndrome [PCOS;

$N=333$; median age (interquartile range) $=30.0$ (9.0) years].

\begin{tabular}{|c|c|}
\hline Variable & $N(\%)$ \\
\hline \multicolumn{2}{|l|}{ Ethnicity } \\
\hline White & $308(92.5)$ \\
\hline Mixed background & $9(2.7)$ \\
\hline Asian or Asian British & $8(2.4)$ \\
\hline Black or Black British & $4(1.2)$ \\
\hline Other ethnic background & $3(0.9)$ \\
\hline Declined to indicate & $1(0.3)$ \\
\hline \multicolumn{2}{|l|}{ Relationship status } \\
\hline Single & $134(40.2)$ \\
\hline Married & $132(39.6)$ \\
\hline Co-habiting & $22(6.6)$ \\
\hline Long-term relationship & $19(5.7)$ \\
\hline Civil-partnership & $12(3.6)$ \\
\hline Engaged & $8(2.4)$ \\
\hline Divorced & $3(0.9)$ \\
\hline Separated & $2(0.6)$ \\
\hline Widowed & $1(0.3)$ \\
\hline \multicolumn{2}{|l|}{ Children } \\
\hline No & $242(72.7)$ \\
\hline Yes & $91(27.3)$ \\
\hline \multicolumn{2}{|l|}{ Education } \\
\hline Undergraduate & $135(40.2)$ \\
\hline College & $107(32.1)$ \\
\hline Postgraduate & $60(18.0)$ \\
\hline Secondary & $26(7.8)$ \\
\hline Doctorate & $5(1.5)$ \\
\hline \multicolumn{2}{|l|}{ Employment } \\
\hline Full-time employment & $210(63.1)$ \\
\hline Part-time employment & $47(14.1)$ \\
\hline Student & $27(8.1)$ \\
\hline House person & $19(5.7)$ \\
\hline Unemployed & $21(6.3)$ \\
\hline Self-employed & $9(2.7)$ \\
\hline \multicolumn{2}{|l|}{ Household income } \\
\hline$\leq £ 39,999$ & $170(51.1)$ \\
\hline$£ 40,000-£ 79,999$ & $137(41.1)$ \\
\hline$\geq £ 80,000$ & $26(7.8)$ \\
\hline
\end{tabular}

All percentage data has been rounded to one decimal place.

also remained statistically significant within each of the two groups (data not shown).

Results of the OLR indicated that Stress, Anxiety, and Depression, as measured by the DASS-21, alongside PCOSQOL domain scores for Mood, Hirsutism, Infertility, and Impact of PCOS were significant predictors of ISI score (all $p$-values $<0.01$; Table 3). Furthermore, we found that the DASS-21 variables were the greatest predictors of ISI score, accounting for the largest proportion of variance in the dependent variable [Stress: Wald $\chi^{2}$ : 87.23, OR: $1.23(1.18,1.29), \mathrm{R}^{2} \mathrm{~N}: 0.05, p<0.0001$; Anxiety: Wald $\chi^{2}$ : 64.06, OR: $1.19(1.14,1.25), \mathrm{R}^{2}{ }_{\mathrm{N}}: 0.03$, $p<0.0001$; Depression: Wald $\chi^{2}$ : 55.5, OR: $1.15(1.11,1.20)$, $\left.\mathrm{R}^{2} \mathrm{~N}: 0.03, p<0.0001\right]$. Finally, no participant characteristic 
significantly influenced the direction or magnitude of the OLR (all $p$-values $>0.2$ ).

\section{DISCUSSION}

To our knowledge, this is the first study to assess the selfreported sleep quality of women with PCOS in the UK during the lockdown/quarantine measures imposed in response to

TABLE 2 | Key outcomes of interest for the study cohort of UK women with polycystic ovary syndrome (PCOS) when split into a dichotomous response regarding the impact of the lockdown measures due to COVID-19 on sleep (reported negative effects vs. no or positive effect).

\begin{tabular}{|c|c|c|c|c|}
\hline $\begin{array}{l}\text { Study variables/ } \\
\text { outcomes }\end{array}$ & $\begin{array}{c}\text { Full study } \\
\text { cohort of } \\
\text { women with } \\
\text { PCOS } \\
(N=333)\end{array}$ & $\begin{array}{c}\text { Negative } \\
\text { impact on } \\
\text { sleep } \\
(N=242)\end{array}$ & $\begin{array}{c}\text { No/positive } \\
\text { impact on } \\
\text { sleep } \\
(N=91)\end{array}$ & $\boldsymbol{P}$ \\
\hline Age (years) & $30.0(9.0)$ & $29.0(9.0)$ & $30.0(10.3)$ & 0.426 \\
\hline $\begin{array}{l}\text { Years Since PCOS } \\
\text { Diagnosis }\end{array}$ & $8.0(9.9)$ & $7.3(9.8)$ & $8.2(12.0)$ & 0.33 \\
\hline Weight (kg) & 93.9 (37.9) & $93.9(37.2)$ & $91.2(37.5)$ & 0.290 \\
\hline $\mathrm{BMI}\left(\mathrm{kg} / \mathrm{m}^{2}\right)$ & $34.8(13.6)$ & $35.0(13.1)$ & $34.0(14.7)$ & 0.32 \\
\hline \multicolumn{5}{|l|}{ PCOSQOL } \\
\hline Total Score & $101.0(60.5)$ & $97.0(59.0)$ & $114.0(65.8)$ & 0.003 \\
\hline Impact of PCOS & $40.0(28.5)$ & $38.0(26.0)$ & 50.5 (30.5) & 0.001 \\
\hline Infertility & $24.0(27.0)$ & $23.0(27.0)$ & 30.5 (26.5) & 0.077 \\
\hline Hirsutism & $16.0(18.0)$ & $16.0(17.0)$ & $17.5(20.3)$ & 0.348 \\
\hline Mood & $17.0(10.0)$ & $16.0(9.0)$ & $21.0(11.0)$ & 0.00 \\
\hline \multicolumn{5}{|l|}{ DASS-21 } \\
\hline Depression & $18.0(17.0)$ & $18.0(16.0)$ & $13.0(16.0)$ & 0.014 \\
\hline Anxiety & $10.0(12.0)$ & $12.0(12.0)$ & $10.0(11.0)$ & 0.09 \\
\hline Stress & $18.0(14.0)$ & $18.0(12.0)$ & $15.0(14.5)$ & 0.007 \\
\hline Insomnia Severity & $12.0(9.5)$ & $14.0(8.0)$ & $7.0(8.0)$ & $<0.001$ \\
\hline Comorbidities & $1.0(2.0)$ & $1.0(2.0)$ & $1.0(3.0)$ & 0.737 \\
\hline
\end{tabular}

Data are presented as median (interquartile range). Between group comparisons performed by independent samples Mann-Whitney U-tests. Insomnia severity as assessed by the validated Insomnia Severity Index (ISI). Significance was set at $p<0.05$. $B M I$, body mass index; PCOSQOL, Polycystic ovary syndrome quality of life questionnaire; DASS-21, Depression, Anxiety, and Stress Scale; $P$, asymptotic two-sided significance. Values in a bold font correspond to $P<0.05$. the COVID-19 pandemic, and explore potential corresponding associations with QoL and depression, anxiety, and stress levels in this female population. Of note, according to the findings of the present study $\sim 73 \%$ of the study participants reported that their sleep quality had worsened since COVID-19 restrictions were imposed. Interestingly, when compared to data from a study in the general population during COVID-19 lockdown measures (62), the prevalence of clinical insomnia based upon the ISI among the women with PCOS of the present study was markedly greater $(\sim 35$ vs. $\sim 10 \%)$. Our findings are in accord with data reported from a web-based study in the Greek general population during the national lockdown due to COVID19 , where $37.6 \%$ of participants (particularly women) scored above the threshold for insomnia based on a relevant validated questionnaire (63). Prior to the COVID-19 pandemic, global estimates for the prevalence of insomnia ranged between 3.9 and 22\% (64), thus these findings suggest that there has been an exacerbation of sleep disturbances (e.g., insomnia) during this pandemic.

The present findings showing that the majority of women with PCOS self-report negative effects upon their sleep during the COVID-19 restrictions highlight insomnia and poor sleep health as a significant problem in this female population. However, it should be noted that it is difficult to determine the magnitude of this problem/change without having relevant baseline assessments before this pandemic. Indeed, due to practical difficulties in studying women with PCOS in representative population-based samples, there is an overall paucity of data on the prevalence of sleep disturbances in this population to allow precise comparisons (65). Notably, one common known sleep disorder in women with PCOS is OSA, with a recent meta-analysis reporting that OSA prevalence in women with PCOS is $35 \%$ (95\% confidence interval: $22.2-48.9 \%$ ) which is further increased in the presence of overweight/obesity (45). Nevertheless, OSA does not appear to be a defining factor for the findings of the present study, since only $1.2 \%$ of participants indicated that they had received a medical diagnosis of OSA, and there were no between group differences for BMI or additional comorbidities (e.g., T2DM) that are often associated with PCOS and OSA (26). Undiagnosed OSA is common in this female population (26), and may also be present among the participants

TABLE 3 | Results from the ordinal logistic regression (OLR) for the study cohort of UK women with polycystic ovary syndrome (PCOS).

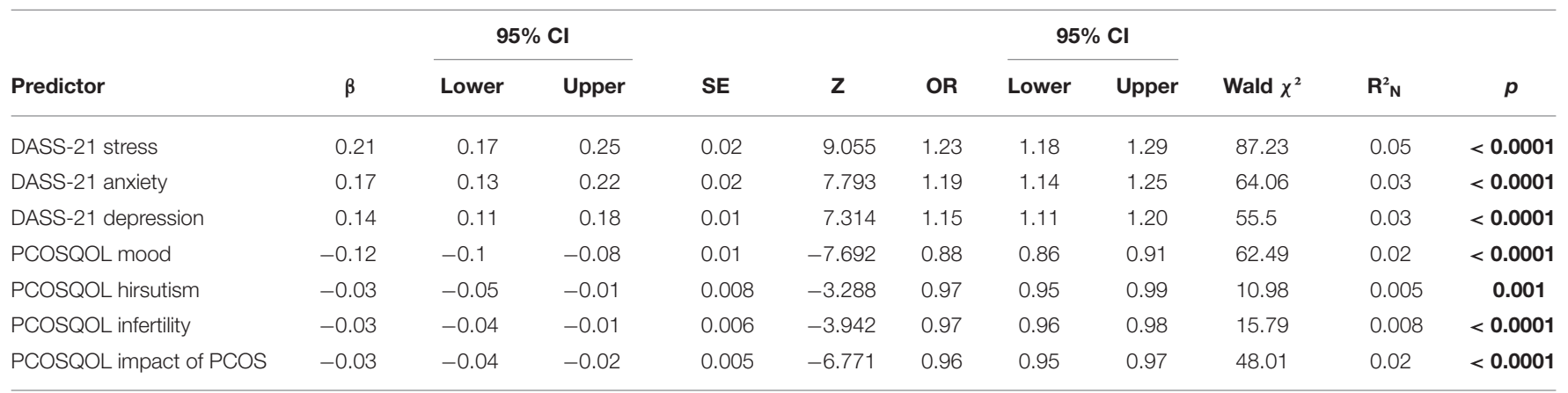

$\mathrm{Cl}$, Confidence Interval; $\beta$, beta-coefficient; SE, Standard Error; OR, Odds ratio; $R^{2} N$, Nagelkerke's pseudo- $R^{2}$; PCOSQOL, Polycystic ovary syndrome quality of life questionnaire; DASS-21, Depression, Anxiety, and Stress Scale (21 item). Values in a bold font correspond to $P<0.05$. 
of this cohort, but whether this could be an underlying factor contributing to the present findings requires further and more targeted research.

Another mechanism contributing to sleep disturbances in this study may relate to increased depression, anxiety, and stress levels. Indeed, depression, anxiety and stress yield the largest beta coefficients and account for the largest amount of variance to ISI scores in the results of the ordinal logistic regression in the present study. Of interest, when the whole study cohort was considered, $51.5 \%$ reported that they had previously received a medical diagnosis of anxiety and/or stress. Whilst it was unclear whether these diagnoses were made prior to, or during the COVID-19 pandemic, additional insight can be gained from the self-reported DASS-21 scores. Based on this validated questionnaire, the prevalence rates of at least mild depression, anxiety, and stress in the study cohort were 80.5, 70.6, and $64.6 \%$, respectively, which are higher than the reported corresponding medical diagnoses. Notably, these are also higher than those reported by a meta-analysis for depression (33.7\%; 95\% CI: $27.5-$ $40.6)$, anxiety (31.9\%; 95\% CI: $27.5-36.7)$, and stress (29.6\%; $95 \%$ CI: 24.3-35.4) in the general population during the COVID19 pandemic (66). Collectively, these data suggest that during the current pandemic, women with PCOS are experiencing a greater psychological burden than the general population, with a markedly higher prevalence. This is in accord with the latest international evidence-based guidance on PCOS, which highlights that, irrespective of a global pandemic, women with PCOS are more likely to experience depression and anxiety (67).

Other reported risk factors for decreased sleep quality during the COVID-19 pandemic are changes to sleep patterns (62), worries about health (68), financial consequences (69), social interactions (70), reduced physical activity (63), and gender with women being reportedly $56 \%$ more likely than men to experience sleep disruption during this pandemic (71). As such, it is plausible that such factors further contribute to a potential multifactorial effect upon an already "at risk" population (72), which clearly exacerbates sleep disruption and may result in reduced QoL. Indeed, the results of the present study support this notion, since women with PCOS who reported negative sleep effects during the COVID-19 pandemic also exhibited reduced QoL (as measured by the PCOSQOL) compared to those without any, or with positive effects on sleep. Based on the corresponding PCOSQOL domains, this association was apparently burdened by the impact of PCOS and affected mood in the study participants. However, what cannot be determined by the present findings is the directional role of sleep disruption in the aforementioned milieu for which further studies are clearly needed.

Interestingly, longitudinal studies have previously reported associations between sleep disorders, anxiety, and depression $(73,74)$, which are known to independently impair QoL (75). Moreover, it has been purported that there is a bidirectional relationship between sleep quality and mental well-being (76), with sleep quality independently predicting the prevalence of anxiety and/or depression, whilst anxiety and depression are also predictors for reduced sleep quality $(42,77)$. Due to the nature of our analysis, the current study is unable to determine the causal direction between sleep and mental well-being. However, it is likely that the COVID-19 pandemic has created an overarching environment which further exacerbates this relationship with the net result being further impairments of mental health and sleep quality leading to reduced QoL. As prior to the COVID19 pandemic, women with PCOS were already recognized as a patient population at an increased risk of anxiety/stress, depression, sleep disorders, and impaired QoL $(18,19)$; the disrupting circumstances of this pandemic mean then that there should be a renewed and heightened focus from healthcare professionals to ensure that adequate support and treatment provision is available to reduce and, where possible, prevent further comorbidity and impaired QoL in these women (16).

\section{Study Limitations}

There are certain limitations which should be acknowledged in the present study. This study relied upon participant selfreport which can lead to a degree of decreased clarity/accuracy in the provided answers, thus inevitably introducing a degree of information bias to the study findings. For example, it is known that individuals tend to over-report their height and under-report their weight (78), an effect which appears to be further exaggerated in individuals with overweight/obesity (79). This may lead to discrepancies in self-reported anthropometric data which, given the key role of metabolic health in PCOS severity/comorbidity (80) and sleep quality (26) may impact to some degree on the study findings. However, this methodology is frequently employed in studies of this nature, whilst validated instruments/questionnaires were utilized to capture key study data of interest. Another study limitation is the lack of comparative baseline data for the study cohort for a period prior to the COVID-19 pandemic. The study survey questions asked about changes in sleep quality due to COVID-19 restriction measures, but without validated baseline measurements, it is not possible to quantify the exact extent to which the corresponding outcomes have been affected. This issue could be addressed to some degree at a follow-up time point when current restrictions have been eased/lifted. To this aim, the relevant prospective follow-up of this study cohort has been planned. Furthermore, although the large sample size in the present study is a distinct strength, it is accompanied by some inherent limitations. For instance, OLR yielded significant $R^{2}$ N for all variables, yet most were of little practical significance; thus, in interpreting these results, the potential fallacy of large sample sizes must be considered (81). Finally, as this is a cross-sectional study, the present findings cannot be used to infer conclusions regarding the temporal/causal relationship between the noted negative impact on sleep and reported levels of depression/stress.

\section{CONCLUSION}

The present study offers a novel insight regarding the selfreported sleep quality of women with PCOS during the COVID19 pandemic lockdown, and how this is associated with QoL and depression, anxiety, and stress levels in this population. Based on our present findings, it is evident that the majority of UK women with PCOS in this study cohort feel that the applied measures imposed in response to the COVID-19 pandemic had a 
negative impact on the quality of their sleep, with high prevalence of insomnia. There is also evidence that those women with PCOS and impaired sleep have greater levels of psychological morbidity (e.g., depression/stress) and reduced QoL. Whilst it appears that the restrictive measures due to COVID-19 have increased this comorbidity burden in these women with PCOS, the exact magnitude of the impact of this global pandemic upon these parameters and the underlying temporal/causal relationship is less clear. Nevertheless, it has previously been reported that during such disease outbreaks, the number of individuals whose mental health is negatively affected can be greater than the number affected by the infection (82), and that the mental health implications and their prevalence can be even more significant than the epidemic itself (83). Another key message to consider based on the present study is that there are certain groups that may remain relatively overlooked despite being particularly vulnerable during this pandemic. Women with PCOS should be considered within these parameters, since they are at increased risk of cardio-metabolic complications which, in turn, may increase the risk of severe COVID-19, whilst they are also susceptible to significant psychological comorbidity which, regardless of COVID-19, may impair their overall well-being.

\section{DATA AVAILABILITY STATEMENT}

The raw data supporting the conclusions of this article will be made available, without undue reservation, by the authors upon reasonable request and where it is ethically acceptable to do so, and does not violate the protection of participants, or other valid ethical, privacy, or security concerns.

\section{ETHICS STATEMENT}

The studies involving human participants were reviewed and approved by Coventry University. The patients/participants provided their online written informed consent to participate in this study.

\section{AUTHOR CONTRIBUTIONS}

CK, LA, GM, JB, IK, and HR developed the study protocol. CK drafted the initial manuscript. CK and CCTC performed the statistical analyses. CK, CCTC, LA, GM, JB, IK, and HR contributed to the literature search, drafted, and/or revised sections of this manuscript. IK and HR supervised the study, combined, edited, and revised all drafts of this manuscript. All authors approved the final manuscript.

\section{ACKNOWLEDGMENTS}

All authors acknowledge and thank all participants of this study and also Verity (the UK PCOS charity) and the PCOS support groups on Facebook for their valuable support to this study. IK and HR also acknowledge and thank the General Charities of the City of Coventry for their support. This paper is also dedicated to all of the healthcare professionals fighting against COVID-19 globally.

\section{REFERENCES}

1. Gupta A, Madhavan MV, Sehgal K, Nair N, Mahajan S, Sehrawat TS, et al. Extrapulmonary manifestations of COVID-19. Nat Med. (2020) 26:1017-32. doi: 10.1038/s41591-020-0968-3

2. Gavriatopoulou M, Korompoki E, Fotiou D, Ntanasis-Stathopoulos I, Psaltopoulou T, Kastritis E, et al. Organ-specific manifestations of COVID-19 infection. Clin Exp Med. (2020) 2020:1-14. doi: 10.1007/s10238-020-00648-x

3. Yuki K, Fujiogi M, Koutsogiannaki S. COVID-19 pathophysiology: a review. Clin Immunol. (2020) 2020:108427. doi: 10.1016/j.clim.2020.108427

4. Nussbaumer-Streit B, Mayr V, Dobrescu AI, Chapman A, Persad E, Klerings I, et al. Quarantine alone or in combination with other public health measures to control COVID-19: a rapid review. Cochrane Database Systematic Rev. (2020) 2020:CD013574. doi: 10.1002/14651858.CD013574

5. Girum T, Lentiro K, Geremew M, Migora B, Shewamare S. Global strategies and effectiveness for COVID-19 prevention through contact tracing, screening, quarantine, and isolation: a systematic review. Trop Med Health. (2020) 48:1-15. doi: 10.1186/s41182-020-00285-w

6. Klonoff DC, Umpierrez GE. COVID-19 in patients with diabetes: risk factors that increase morbidity. Metabolism. (2020) 2020:154224. doi: 10.1016/j.metabol.2020.154224

7. Li X, Xu S, Yu M, Wang K, Tao Y, Zhou Y, et al. Risk factors for severity and mortality in adult COVID-19 inpatients in Wuhan. J Allergy Clin Immunol. (2020) 2020:6. doi: 10.1016/j.jaci.2020.04.006

8. Zuin M, Rigatelli G, Zuliani G, Rigatelli A, Mazza A, Roncon L. Arterial hypertension and risk of death in patients with COVID-19 infection: systematic review and meta-analysis. J Infection. (2020) 2020:59. doi: 10.1016/j.jinf.2020.03.059

9. Hamer M, Gale CR, Kivimäki M, Batty GD. Overweight, obesity, and risk of hospitalization for COVID-19: a community-based cohort study of adults

in the United Kingdom. Proc Natl Acad Sci USA. (2020) 117:21011-3. doi: 10.1073/pnas.2011086117

10. Hamer M, Gale CR, Batty GD. Diabetes, glycaemic control, and risk of COVID-19 hospitalisation: population-based, prospective cohort study. Metabolism. (2020) 112:154344. doi: 10.1016/j.metabol.2020.154344

11. Iaccarino G, Grassi G, Borghi C, Ferri C, Salvetti M, Volpe M. Age and multimorbidity predict death among COVID-19 patients: results of the SARSRAS study of the Italian Society of Hypertension. Hypertension. (2020) 76:366-72. doi: 10.1161/HYPERTENSIONAHA.120.15324

12. McGurnaghan SJ, Weir A, Bishop J, Kennedy S, Blackbourn LA, McAllister DA, et al. Risks of and risk factors for COVID-19 disease in people with diabetes: a cohort study of the total population of Scotland. Lancet Diabetes Endocrinol. (2020) 9:82-93. doi: 10.1016/S2213-8587(20)30405-8

13. Acheampong DO, Barffour IK, Boye A, Aninagyei E, Ocansey S, Morna MT. Male predisposition to severe COVID-19: review of evidence and potential therapeutic prospects. Biomed Pharmacotherapy. (2020) 2020:110748. doi: 10.1016/j.biopha.2020.110748

14. Haitao T, Vermunt J, Abeykoon J, Ghamrawi R, Gunaratne M, Jayachandran M, et al. COVID-19 and sex differences: mechanisms and biomarkers. Mayo Clin Proc. (2020) 2020:24. doi: 10.1016/j.mayocp.2020.07.024

15. Ding T, Hardiman PJ, Petersen I, Baio G. Incidence and prevalence of diabetes and cost of illness analysis of polycystic ovary syndrome: a Bayesian modelling study. Hum Reprod. (2018) 33:1299-306. doi: 10.1093/humrep/dey093

16. Kyrou I, Karteris E, Robbins T, Chatha K, Drenos F, Randeva HS. Polycystic ovary syndrome (PCOS) and COVID-19: an overlooked female patient population at potentially higher risk during the COVID-19 pandemic. $B M C$ Med. (2020) 18:1-10. doi: 10.1186/s12916-020-01697-5

17. Moin ASM, Sathyapalan T, Atkin SL, Butler AE. Renin-Angiotensin System overactivation in polycystic ovary syndrome, a risk for SARS-CoV-2 infection? Metabol Open. (2020) 7:100052. doi: 10.1016/j.metop.2020.100052 
18. Boyle JA, Cunningham J, O’Dea K, Dunbar T, Norman RJ. Prevalence of polycystic ovary syndrome in a sample of Indigenous women in Darwin, Australia. Medical J Australia. (2012) 196:62-6. doi: 10.5694/mja11. 10553

19. Kyrou I, Weickert MO, Randeva HS. Diagnosis and management of polycystic ovary syndrome (PCOS). Endocrinol Diabetes. (2015) 13:99-113. doi: 10.1007/978-1-4471-2789-5_13

20. Kyritsi EM, Dimitriadis GK, Kyrou I, Kaltsas G, Randeva HS. PCOS remains a diagnosis of exclusion: a concise review of key endocrinopathies to exclude. Clin Endocrinol. (2017) 86:1-6. doi: 10.1111/cen.13245

21. ESHRE TR, Group A-SPCW. Revised 2003 consensus on diagnostic criteria and long-term health risks related to polycystic ovary syndrome. Fertil Steril. (2004) 81:19-25. doi: 10.1016/j.fertnstert.2003.10.004

22. Lim SS, Davies M, Norman RJ, Moran L. Overweight, obesity and central obesity in women with polycystic ovary syndrome: a systematic review and meta-analysis. Hum Reprod Update. (2012) 18:618-37. doi: 10.1093/humupd/dms030

23. Stepto NK, Cassar S, Joham AE, Hutchison SK, Harrison CL, Goldstein RF, et al. Women with polycystic ovary syndrome have intrinsic insulin resistance on euglycaemic-hyperinsulaemic clamp. Hum Reprod. (2013) 28:777-84. doi: 10.1093/humrep/des463

24. Kyrou I, Randeva HS, Tsigos C, Kaltsas G, Weickert MO. Clinical Problems Caused by Obesity. In: Feingold KR, Anawalt B, Boyce A, Chrousos G, de Herder WW, Dhatariya K, Dungan K, Grossman A, Hershman JM, Hofland J, Kalra S, Kaltsas G, Koch C, Kopp P, Korbonits M, Kovacs CS, Kuohung W, Laferrère B, McGee EA, McLachlan R, Morley JE, New M, Purnell M, Sahay R, Singer F, Stratakis CA, Trence DL, and Wilson DP, editors, Endotext. South Dartmouth, MA: MDText.com, Inc. (2018). Available online at: https://www. ncbi.nlm.nih.gov/books/NBK278973/

25. Randeva HS, Tan BK, Weickert MO, Lois K, Nestler JE, Sattar N, et al. Cardiometabolic aspects of the polycystic ovary syndrome. Endocr Rev. (2012) 33:812-41. doi: 10.1210/er.2012-1003

26. Kahal H, Kyrou I, Tahrani AA, Randeva HS. Obstructive sleep apnoea and polycystic ovary syndrome: a comprehensive review of clinical interactions and underlying pathophysiology. Clin Endocrinol. (2017) 87:3139. doi: $10.1111 /$ cen. 13392

27. Moran LJ, Misso ML, Wild RA, Norman RJ. Impaired glucose tolerance, type 2 diabetes and metabolic syndrome in polycystic ovary syndrome: a systematic review and meta-analysis. Hum Reprod Update. (2010) 16:347-63. doi: 10.1093/humupd/dmq001

28. Barry JA, Kuczmierczyk AR, Hardiman PJ. Anxiety and depression in polycystic ovary syndrome: a systematic review and meta-analysis. Hum Reprod. (2011) 26:2442-51. doi: 10.1093/humrep/der197

29. Karjula S, Morin-Papunen L, Auvinen J, Ruokonen A, Puukka K, Franks S, et al. Psychological distress is more prevalent in fertile age and premenopausal women with PCOS symptoms: 15-year follow-up. J Clin Endocrinol Metabol. (2017) 102:1861-9. doi: 10.1210/jc.2016-3863

30. Tay CT, Teede HJ, Hill B, Loxton D, Joham AE. Increased prevalence of eating disorders, low self-esteem, and psychological distress in women with polycystic ovary syndrome: a community-based cohort study. Fertil Steril. (2019) 112:353-61. doi: 10.1016/j.fertnstert.2019.03.027

31. Teede H, Deeks A, Moran L. Polycystic ovary syndrome: a complex condition with psychological, reproductive and metabolic manifestations that impacts on health across the lifespan. BMC Med. (2010) 8:41. doi: 10.1186/1741-7015-8-41

32. Jedel E, Waern M, Gustafson D, Landen M, Eriksson E, Holm G, et al. Anxiety and depression symptoms in women with polycystic ovary syndrome compared with controls matched for body mass index. Hum Reprod. (2010) 25:450-6. doi: 10.1093/humrep/dep384

33. Podfigurna-Stopa A, Luisi S, Regini C, Katulski K, Centini G, Meczekalski B, et al. Mood disorders and quality of life in polycystic ovary syndrome. Gynecol Endocrinol. (2015) 31:431-4. doi: 10.3109/09513590.2015.1009437

34. Duan L, Zhu G. Psychological interventions for people affected by the COVID-19 epidemic. Lancet Psychiatry. (2020) 7:300-2. doi: 10.1016/S2215-0366(20)30073-0

35. Rubin GJ, Wessely S. The psychological effects of quarantining a city. BMJ. (2020) 368:313. doi: 10.1136/bmj.m313
36. Ayittey FK, Ayittey MK, Chiwero NB, Kamasah JS, Dzuvor C. Economic impacts of Wuhan 2019-nCoV on China and the world. J Medical Virol. (2020) 92:473-5. doi: 10.1002/jmv.25706

37. Wu K, Chan SK, Ma TM. Posttraumatic stress, anxiety, and depression in survivors of severe acute respiratory syndrome (SARS). J Traumatic Stress. (2005) 18:39-42. doi: 10.1002/jts.20004

38. Rajkumar RP. COVID-19 and mental health: a review of the existing literature. Asian J Psychiatry. (2020) 2020:102066. doi: 10.1016/j.ajp.2020.102066

39. Shen L, van Schie J, Ditchburn G, Brook L, Bei B. Positive and negative emotions: differential associations with sleep duration and quality in adolescents. J Youth Adolesc. (2018) 47:2584-95. doi: 10.1007/s10964-018-0899-1

40. Ford DE, Kamerow DB. Epidemiologic study of sleep disturbances and psychiatric disorders: an opportunity for prevention? JAMA. (1989) 262:147984. doi: 10.1001/jama.262.11.1479

41. Breslau N, Roth T, Rosenthal L, Andreski P. Sleep disturbance and psychiatric disorders: a longitudinal epidemiological study of young adults. Biol Psychiatry. (1996) 39:411-8. doi: 10.1016/0006-3223(95)00188-3

42. Kaneita Y, Yokoyama E, Harano S, Tamaki T, Suzuki H, Munezawa T, et al. Associations between sleep disturbance and mental health status: a longitudinal study of Japanese junior high school students. Sleep Med. (2009) 10:780-6. doi: 10.1016/j.sleep.2008.06.014

43. Harvey AG, Murray G, Chandler RA, Soehner A. Sleep disturbance as transdiagnostic: consideration of neurobiological mechanisms. Clin Psychol Rev. (2011) 31:225-35. doi: 10.1016/j.cpr.2010.04.003

44. Kutenaee MA, Amirjani S, Asemi Z, Taghavi S-A, Allan H, Kamalnadian S$\mathrm{N}$, et al. The impact of depression, self-esteem, and body image on sleep quality in patients with PCOS: a cross-sectional study. Sleep Breathing. (2020) 24:1027-34. doi: 10.1007/s11325-019-01946-9

45. Kahal H, Kyrou I, Uthman OA, Brown A, Johnson S, Wall PD, et al. The prevalence of obstructive sleep apnoea in women with polycystic ovary syndrome: a systematic review and meta-analysis. Sleep Breathing. (2020) 24:339-50. doi: 10.1007/s11325-019-01835-1

46. Moran L, March W, Whitrow M, Giles L, Davies M, Moore V. Sleep disturbances in a community-based sample of women with polycystic ovary syndrome. Hum Reprod. (2015) 30:466-72. doi: 10.1093/humrep/deu318

47. Kahal H, Tahrani AA, Kyrou I, Dimitriadis GK, Kimani PK, Barber $\mathrm{TM}$, et al. The relationship between obstructive sleep apnoea and quality of life in women with polycystic ovary syndrome: a cross-sectional study. Therapeutic Adv Endocrinol Metabol. (2020) 11:2042018820906689. doi: $10.1177 / 2042018820906689$

48. Williams S, Sheffield D, Knibb RC. The Polycystic Ovary Syndrome Quality of Life scale (PCOSQOL): development and preliminary validation. Health Psychol Open. (2018) 5:2055102918788195. doi: 10.1177/2055102918788195

49. Kitzinger C, Willmott J. "The thief of womanhood": women's experience of polycystic ovarian syndrome. Soc Sci Med. (2002) 54:349-61. doi: 10.1016/S0277-9536(01)00034-X

50. Williams S, Sheffield D, Knibb RC. A snapshot of the lives of women with polycystic ovary syndrome: a photovoice investigation. J Health Psychol. (2016) 21:1170-82. doi: 10.1177/1359105314547941

51. Williams S, Sheffield D, Knibb RC. "Everything's from the inside out with PCOS": exploring women's experiences of living with polycystic ovary syndrome and co-morbidities through Skype ${ }^{\mathrm{TM}}$ interviews. Health Psychol Open. (2015) 2:2055102915603051. doi: 10.1177/2055102915603051

52. Lovibond PF, Lovibond SH. The structure of negative emotional states: comparison of the Depression Anxiety Stress Scales (DASS) with the Beck Depression and Anxiety Inventories. Behav Res Therapy. (1995) 33:335-43. doi: 10.1016/0005-7967(94)00075-U

53. Morin CM. Insomnia Severity Index (ISI) [Database record]. APA PsycTests (1993). doi: 10.1037/t07115-000

54. Bastien $\mathrm{CH}$, Vallières A, Morin CM. Validation of the Insomnia Severity Index as an outcome measure for insomnia research. Sleep Med. (2001) 2:297-307. doi: 10.1016/S1389-9457(00)00065-4

55. Morin CM, Belleville G, Bélanger L, Ivers H. The Insomnia Severity Index: psychometric indicators to detect insomnia cases and evaluate treatment response. Sleep. (2011) 34:601-8. doi: 10.1093/sleep/34.5.601 
56. R Core Team. R: A Language and Environment for Statistical Computing (Computer Software). Vienna: R Foundation for Statistical Computing (2018). Available online at: https://cran.r-project.org/

57. Fox J, Weisberg S, Price B, Adler D, Bates D, Baud-Bovy G. Car: Companion to Applied Regression. R Package Version 3.0-2. (2018). Available online at: https://cran.r-projectorg/web/packages/car (accessed May 19, 2021).

58. Ripley B, Venables W, Bates DM, Hornik K, Gebhardt A, Firth D. MASS: Support Functions and Datasets for Venables and Ripley's MASS. (2018). Available online at: https://cran.r-project.org/package=MASS (accessed May 19, 2021).

59. Hosmer Jr DW, Lemeshow S, Sturdivant RX. Applied Logistic Regression. John Wiley \& Sons (2013). doi: 10.1002/9781118548387

60. Cox D, Snell E. Special Logistic Analyses. Analysis of Binary Data. 2nd ed. London: Chapman and Hall (1989). p. 26-105.

61. Nagelkerke NJ. A note on a general definition of the coefficient of determination. Biometrika. (1991) 78:691-2. doi: 10.1093/biomet/78.3.691

62. Gupta R, Grover S, Basu A, Krishnan V, Tripathi A, Subramanyam A, et al. Changes in sleep pattern and sleep quality during COVID-19 lockdown. Ind J Psychiatry. (2020) 62:370. doi: 10.4103/psychiatry.IndianJPsychiatry_523_20

63. Voitsidis P, Gliatas I, Bairachtari V, Papadopoulou K, Papageorgiou G, Parlapani E, et al. Insomnia during the COVID-19 pandemic in a Greek population. Psychiatry Res. (2020) 2020:113076. doi: 10.1016/j.psychres.2020.113076

64. Kay-Stacey M, Attarian H. Advances in the management of chronic insomnia. BMJ. (2016) 354:i2123. doi: 10.1136/bmj.i2123

65. Fernandez RC, Moore VM, Van Ryswyk EM, Varcoe TJ, Rodgers RJ, March WA, et al. Sleep disturbances in women with polycystic ovary syndrome: prevalence, pathophysiology, impact and management strategies. Nat Sci Sleep. (2018) 10:45. doi: 10.2147/NSS.S127475

66. Salari N, Hosseinian-Far A, Jalali R, Vaisi-Raygani A, Rasoulpoor S, Mohammadi $M$, et al. Prevalence of stress, anxiety, depression among the general population during the COVID-19 pandemic: a systematic review and meta-analysis. Globalization Health. (2020) 16:1-11. doi: 10.1186/s12992-020-00589-w

67. Teede HJ, Misso ML, Costello MF, Dokras A, Laven J, Moran L, et al. Recommendations from the international evidence-based guideline for the assessment and management of polycystic ovary syndrome. Hum Reprod. (2018) 33:1602-18. doi: 10.1093/humrep/dey256

68. Casagrande M, Favieri F, Tambelli R, Forte G. The enemy who sealed the world: effects quarantine due to the COVID-19 on sleep quality, anxiety, and psychological distress in the Italian population. Sleep Med. (2020) 2020:3576805. doi: 10.2139/ssrn.3576805

69. Mandelkorn U, Genzer S, Choshen-Hillel S, Reiter J, Meira e Cruz M, Hochner $\mathrm{H}$, et al. Escalation of sleep disturbances amid the COVID-19 pandemic: a cross-sectional international study. J Clin Sleep Med. (2020) 2020:jcsm.8800. doi: $10.5664 / \mathrm{jcsm} .8800$

70. Altena E, Baglioni C, Espie CA, Ellis J, Gavriloff D, Holzinger B, et al. Dealing with sleep problems during home confinement due to the COVID-19 outbreak: practical recommendations from a task force of the European CBT-I Academy. J Sleep Res. (2020) 2020:e13052. doi: 10.1111/jsr. 13052

71. Ara T, Rahman M, Hossain M, Ahmed A. Identifying the associated risk factors of sleep disturbance during the COVID-19 lockdown in Bangladesh: a web-based survey. Front Psychiatry. (2020) 11:966. doi: 10.3389 /fpsyt.2020.580268

72. Sam S, Ehrmann DA. Pathogenesis and consequences of disordered sleep in PCOS. Clin Med Insights. (2019) 13:1179558119871269. doi: $10.1177 / 1179558119871269$

73. Fichter MM, Kohlboeck G, Quadflieg N, Wyschkon A, Esser G. From childhood to adult age: 18-year longitudinal results and prediction of the course of mental disorders in the community. Soc Psychiatry Psychiatric Epidemiol. (2009) 44:792-803. doi: 10.1007/s00127-009-0501-y

74. Baglioni C, Battagliese G, Feige B, Spiegelhalder K, Nissen C, Voderholzer $\mathrm{U}$, et al. Insomnia as a predictor of depression: a meta-analytic evaluation of longitudinal epidemiological studies. J Affective Disord. (2011) 135:10-9. doi: 10.1016/j.jad.2011.01.011

75. Zeitlhofer J, Schmeiser-Rieder A, Tribl G, Rosenberger A, Bolitschek J, Kapfhammer G, et al. Sleep and quality of life in the Austrian population. Acta Neurol Scand. (2000) 102:249-57. doi: 10.1034/j.1600-0404.2000.102004249.x

76. Alvaro PK, Roberts RM, Harris JK. A systematic review assessing bidirectionality between sleep disturbances, anxiety, and depression. Sleep. (2013) 36:1059-68. doi: 10.5665/sleep.2810

77. Jansson-Fröjmark M, Lindblom K. A bidirectional relationship between anxiety and depression, and insomnia? A prospective study in the general population. $J$ Psychosomatic Res. (2008) 64:443-9. doi: 10.1016/j.jpsychores.2007.10.016

78. Gorber SC, Tremblay M, Moher D, Gorber B. A comparison of direct vs. self-report measures for assessing height, weight and body mass index: a systematic review. Obesity Rev. (2007) 8:307-26. doi: 10.1111/j.1467-789X.2007.00347.x

79. Nawaz H, Chan W, Abdulrahman M, Larson D, Katz DL. Self-reported weight and height: implications for obesity research. Am J Prev Med. (2001) 20:294-8. doi: 10.1016/S0749-3797(01)00293-8

80. Legro RS. Obesity and PCOS: implications for diagnosis and treatment. Semin Reprod Med. (2012) 32:1328878. doi: 10.1055/s-0032-1328878

81. Lantz B. The large sample size fallacy. Scand J Caring Sci. (2013) 27:487-92. doi: $10.1111 / j .1471-6712.2012 .01052 . x$

82. Reardon S. Ebola's mental-health wounds linger in Africa: health-care workers struggle to help people who have been traumatized by the epidemic. Nature. (2015) 519:13-5. doi: 10.1038/519013a

83. Shigemura J, Ursano RJ, Morganstein JC, Kurosawa M, Benedek DM. Public responses to the novel 2019 coronavirus (2019-nCoV) in Japan: mental health consequences and target populations. Psychiatry Clin Neurosci. (2020) 74:281. doi: $10.1111 / \mathrm{pcn} .12988$

Conflict of Interest: The authors declare that the research was conducted in the absence of any commercial or financial relationships that could be construed as a potential conflict of interest.

Copyright $\odot 2021$ Kite, Atkinson, McGregor, Clark, Brown, Kyrou and Randeva. This is an open-access article distributed under the terms of the Creative Commons Attribution License (CC BY). The use, distribution or reproduction in other forums is permitted, provided the original author(s) and the copyright owner(s) are credited and that the original publication in this journal is cited, in accordance with accepted academic practice. No use, distribution or reproduction is permitted which does not comply with these terms. 